\title{
THE HISTOPATHOLOGY OF PSORIASIS
}

\author{
G. DE ROSA, C. MIGNOGNA \\ Department of Biomorphological and Functional Sciences, Pathology Section, “Federico II” University of Naples, Italy
}

\begin{abstract}
SUMMARY
Psoriasis is a common, chronic, relapsing, papulo-squamous dermatitis, with overlying silvery scales. The scalp, sacral region, and extensor surfaces of extremity are commonly involved, even ifflexural and intertriginous areas may be affected in the so-called "inverse psoriasis". Involvement of nails is frequent. Oral lesions (geographic stomatitis and/or glossitis) are commonly described. 5-8\% of psoriatic patients develop arthritis. Interphalangeal joints are characteristically involved, but large joints are also affected.

From a histological point of view, psoriasis is a dynamic dermatosis that changes during the evolution of an individual lesion; we can classify it in an early stage, advanced stage, and later lesions. Lesions are usually diagnostic only in early stages or near the margin of advancing plaques. Munro microabscesses and Kogoj micropustoles are diagnostic clues of psoriasis, but they aren't always present. All other features can be found in numerous eczematous dermatitis.
\end{abstract}

Key words: Psoriasis, histopathology, immunohistochemistry

\section{PRESENTATION}

Normal skin is constituted of epidermidis, epidermal basement membrane, papillary and reticular dermis, with related adnexa, and subcutaneous fat. Epidermis shows an ordered maturation of keratinocytes from the basal (germinative) layer, toward the spinosum, the granular cell layer up to the keratinized layer. It is very important to remember that a considerable morphological variation exists in normal skin, due to the topographic area, and to physiological variation depending from hormonal balance, sex, and age.

The interpretation of skin biopsies by a Dermatopathologyst for non-neoplastic pathologies requires a first discrimination between major tissue reaction patterns. In this occasion we want to highlight the psoriasiform reaction pattern, shared by an eterogeneous group of dermatologic diseases (morphologically defined as epidermal hyperplasia with elongation of the rete ridges, usually in a regular manner) of which psoriasis is the prototype.

Corresponding author:

Chiara Mignogna

Via Torrione S. Martino 43

80129 Napoli, Italy

E-mail: mignogna@libero.it
Psoriasis is a common chronic, relapsing, papulosquamous dermatitis, characterized by an epidermis covered by silvery scales (1).

The scalp, sacral region, and extensor surfaces of extremity are commonly involved, even if flexural and intertriginous areas may be affected in the socalled "inverse psoriasis". Involvement of nails is frequent. Oral lesions (geographic stomatitis and/or glossitis) are commonly described.

Between 5 to $8 \%$ of psoriatic patients develop arthritis, which presents itself according to one of

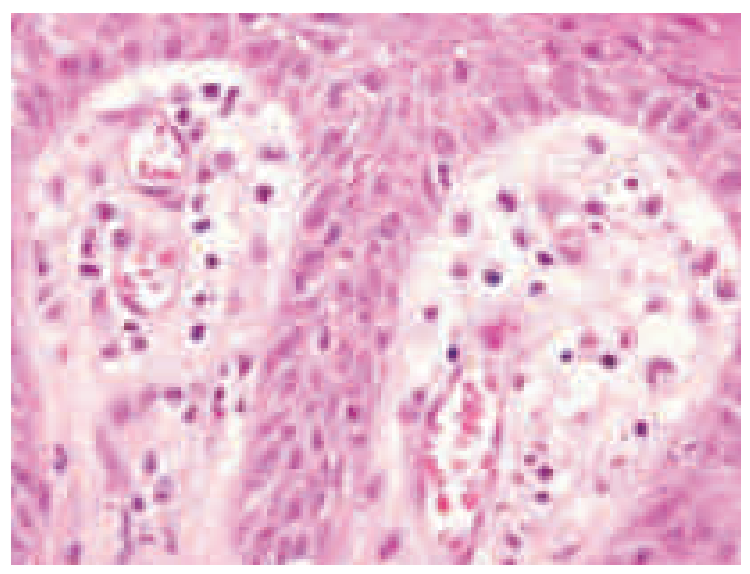

Figure 1 - Vessels dilated and tortuous, with light edema and lymphocytic infiltrate. 
five varieties, with frequent overlapping forms:

1) Peripheral type;

2) Peripheral symmetric polyarthritis;

3) Asymmetrical joint type;

4) Spondiloarthopathy;

5) Mutilating peripheral type.

Psoriasis is a dynamic dermatosis with morphological changes during the evolution of an individual lesion.

The early stage consists in elongation and dilatation of blood vessels of the papillary derma, with associated aedema and lymphocytic infiltrate (perivascular cuffing). Vessels are dilated and tortuous, with some neutrophils in their lumen. Lymphocytes and neutrophils emerge from the vessel reaching the epidermis ("squirting" papilla). Rare erythrocytes extravasated may be found. Epidermis during this phase, is quite normal (Fig. 1).

Shortly after, there is a thickening of epidermis with loss of granular cell layer and formations of mounds of parakeratosis, which is thought to result from a markedly shortened cellular turnover time. Keratinocytes proliferate and mature rapidly, so that terminal differentiation is incomplete. Thus, squamous keratinocytes aberrantly retain intact nuclei and release few extracellular lipids that normally cement adhesion of corneocytes. The resulting poorly adherent stratum corneum leads to the characteristic scales or flakes of psoriasis lesions (2). Scattered neutrophils are seen at the edge of mounds of parakeratosis: they represents the earliest manifestation of Munro microabscesses (see below).

The advanced stage is characterized by regular acanthosis (thickening of stratum spinosum) and epidermal "psoriasiform hyperplasia" with regular

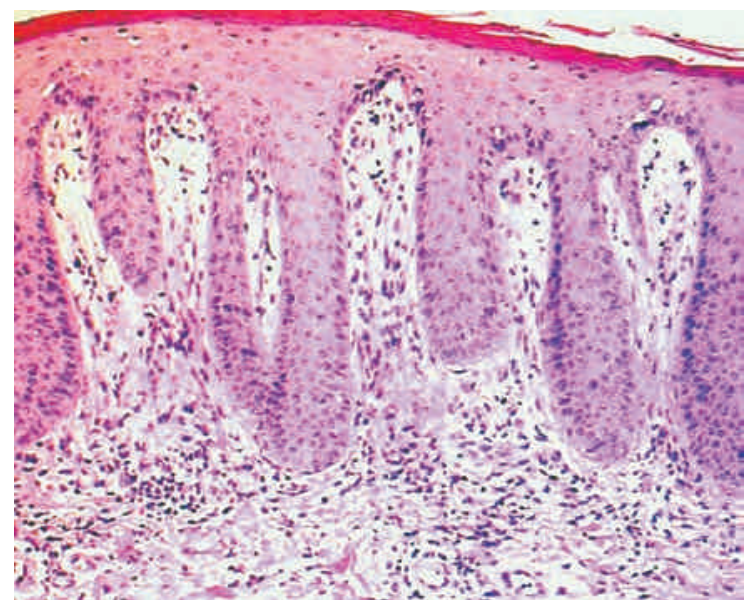

Figure 2 - Epidermal psoriasiform hyperplasia.

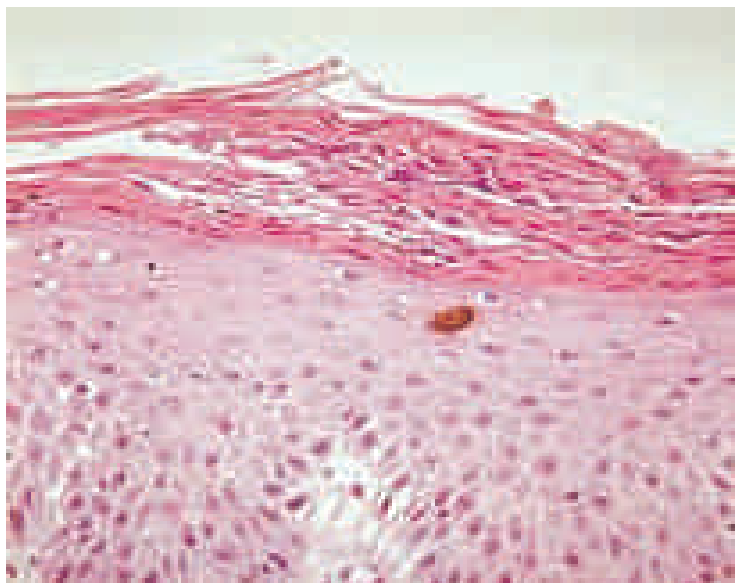

Figure 3 - Munro microabscesses.

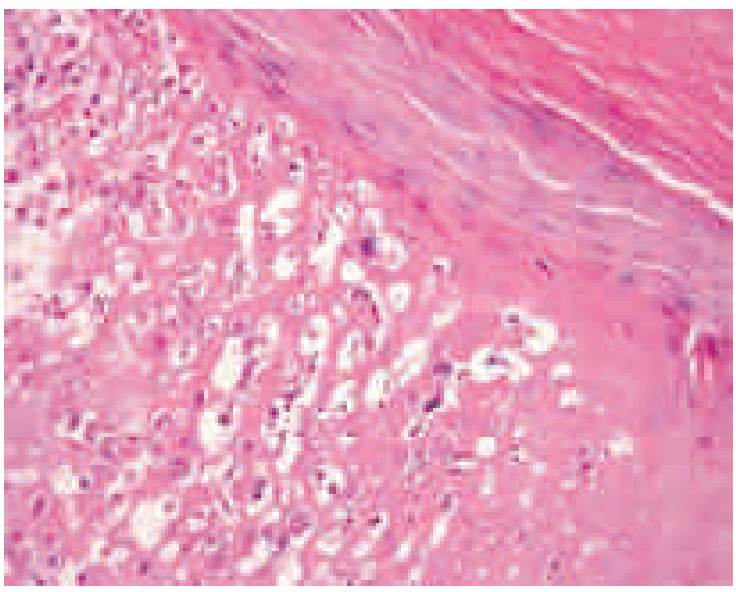

Figure 4 - Spongiform pustule of Kogoj.

elongation of the rete ridges and thinning of suprapapillary plates (Fig 2).

Mitotic activity is often quite marked, a further indicator of the hyperproliferative nature of this condition.

Parakeratosis become confluent, with loss of granular layer. There is transmigration of inflammatory cells through epidermis into parakeratotic scale resulting in intracorneal collections of neutrophils, the so called "Munro microabscesses" (Fig. 3). Similar accumulation in the stratum spinosum are defined as "spongiform pustule of Kogoj" (Fig. 4). When subcorneal abscesses are prominent the disease is designated as pustular psoriasis (Fig. 5). Dermal inflammatory infiltrate is heavier than in early lesions, it is composed by $\mathrm{T}$ lymphocytes, containg a few Langherhan cells with occasional neutrophils.

Under the epidermal basement membrane, macrophages CD11c positive are present (3). In 


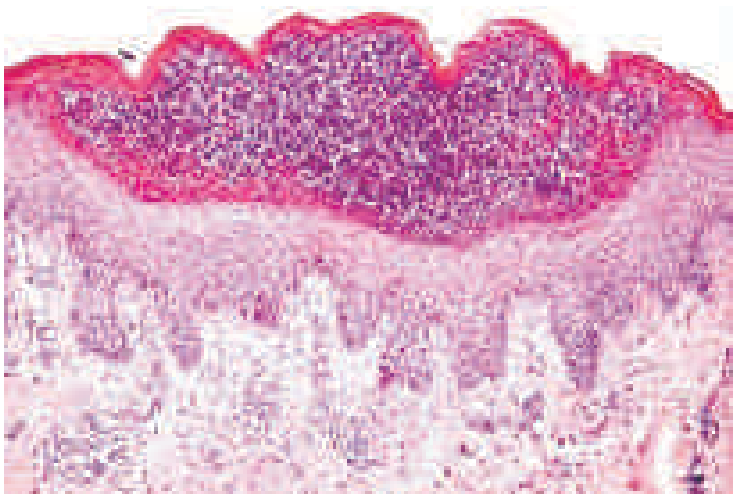

Figure 5 - Pustolar psoriasis.

addition to neutrophils, T lymphocytes are also found interspersed between keratinocytes throughout the epidermis and in larger quantities in the dermis. With immunohistochemical analysis it has been shown that epidermal lymphocytes are chiefly CD8+ $\mathrm{T}$ cells, while dermic lymphocytes are a mixture of CD4+ and CD8+ T cells, with a CD4+ predominance, similar to that seen in peripheral blood (2).

Later lesions there is orthokeratosis, an intact granular layer and mild exocytosis of inflammatory cells.

Munro microabscesses and Kogoj micropustoles are diagnostic clues of psoriasis, but they are not always present. All other features can be found in numerous eczematous dermatitis, such as allergic contact dermatitis and atopic dermatitis. However, in these lesions spongiosis and "oozing" (presence of coagulated serum in cornified layer) are marked. Moreover, in allergic contact dermatitis there is a characteristic eosinophilic infiltrate which is normally absent in psoriasis.

Later lesions must be distinguished from Lichen simplex chronicus that in contrast to psoriasis shows an evident granular layer, marked acanthosis and dermic fibrosis. Seborreic dermatitis could be hardly differentiated from psoriasis by the evident spongiosis, follicular parakeratosis and irregular acanthosis. PAS and Gram stains are helpful to identify microorganisms, in cases of bacterial impetigo, candidiasis and pustolar dermatophytosis.

\section{REFERENCES}

1. Barr RJ, Young EM Jr. Psoriasiform and related papulosquamous disorders. J Cutan Pathol 1985; 12: 412 425

2. Krueger JG, Bowcock A. Psoriasis pathophysiology: current concepts of pathogenesis. Ann Rheum Dis 2005; 64 (suppl) 30-36.

3. Weedon D, Strutton J. The psoriasiform reaction pattern. In: David Weedon Skin Pathology 2th ed Churchill Livingstone London 2002; 75-83. 